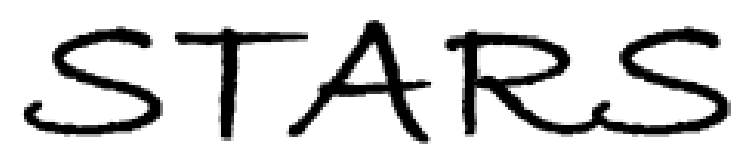

University of Central Florida

STARS

$1-1-2009$

\title{
Quantum chemistry of quantum dots: Effects of ligands and oxidation
}

Talgat M. Inerbaev

University of Central Florida

Artëm E. Masunov

University of Central Florida

Saiful I. Khondaker

University of Central Florida

Alexandra Dobrinescu

University of Central Florida

Andrei-Valentin Plamada

University of Central Florida

Find similar works at: https://stars.library.ucf.edu/facultybib2000

University of Central Florida Libraries http://library.ucf.edu

See next page for additional authors

This Article is brought to you for free and open access by the Faculty Bibliography at STARS. It has been accepted for inclusion in Faculty Bibliography 2000s by an authorized administrator of STARS. For more information, please contactSTARS@ucf.edu.

\section{Recommended Citation}

Inerbaev, Talgat M.; Masunov, Artëm E.; Khondaker, Saiful I.; Dobrinescu, Alexandra; Plamada, AndreiValentin; and Kawazoe, Yoshiyuki, "Quantum chemistry of quantum dots: Effects of ligands and oxidation" (2009). Faculty Bibliography 2000s. 1657.

https://stars.library.ucf.edu/facultybib2000/1657

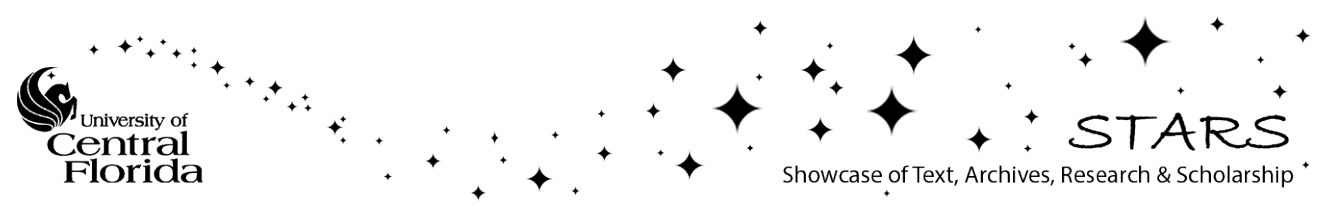




\section{Authors}

Talgat M. Inerbaev, Artëm E. Masunov, Saiful I. Khondaker, Alexandra Dobrinescu, Andrei-Valentin Plamada, and Yoshiyuki Kawazoe 


\section{Quantum chemistry of quantum dots: Effects of ligands and oxidation}

Cite as: J. Chem. Phys. 131, 044106 (2009); https://doi.org/10.1063/1.3135193

Submitted: 15 January 2009 . Accepted: 27 April 2009. Published Online: 23 July 2009

Talgat M. Inerbaev, Artëm E. Masunov, Saiful I. Khondaker, Alexandra Dobrinescu, Andrei-Valentin Plamadă, and Yoshiyuki Kawazoe

\section{ARTICLES YOU MAY BE INTERESTED IN}

Electron-electron and electron-hole interactions in small semiconductor crystallites: The size dependence of the lowest excited electronic state

The Journal of Chemical Physics 80, 4403 (1984); https://doi.org/10.1063/1.447218

Size-dependent band gap of colloidal quantum dots

Journal of Applied Physics 99, 013708 (2006); https://doi.org/10.1063/1.2158502

Inorganic-ligand exchanging time effect in $\mathrm{PbS}$ quantum dot solar cell

Applied Physics Letters 109, 063901 (2016); https://doi.org/10.1063/1.4960645

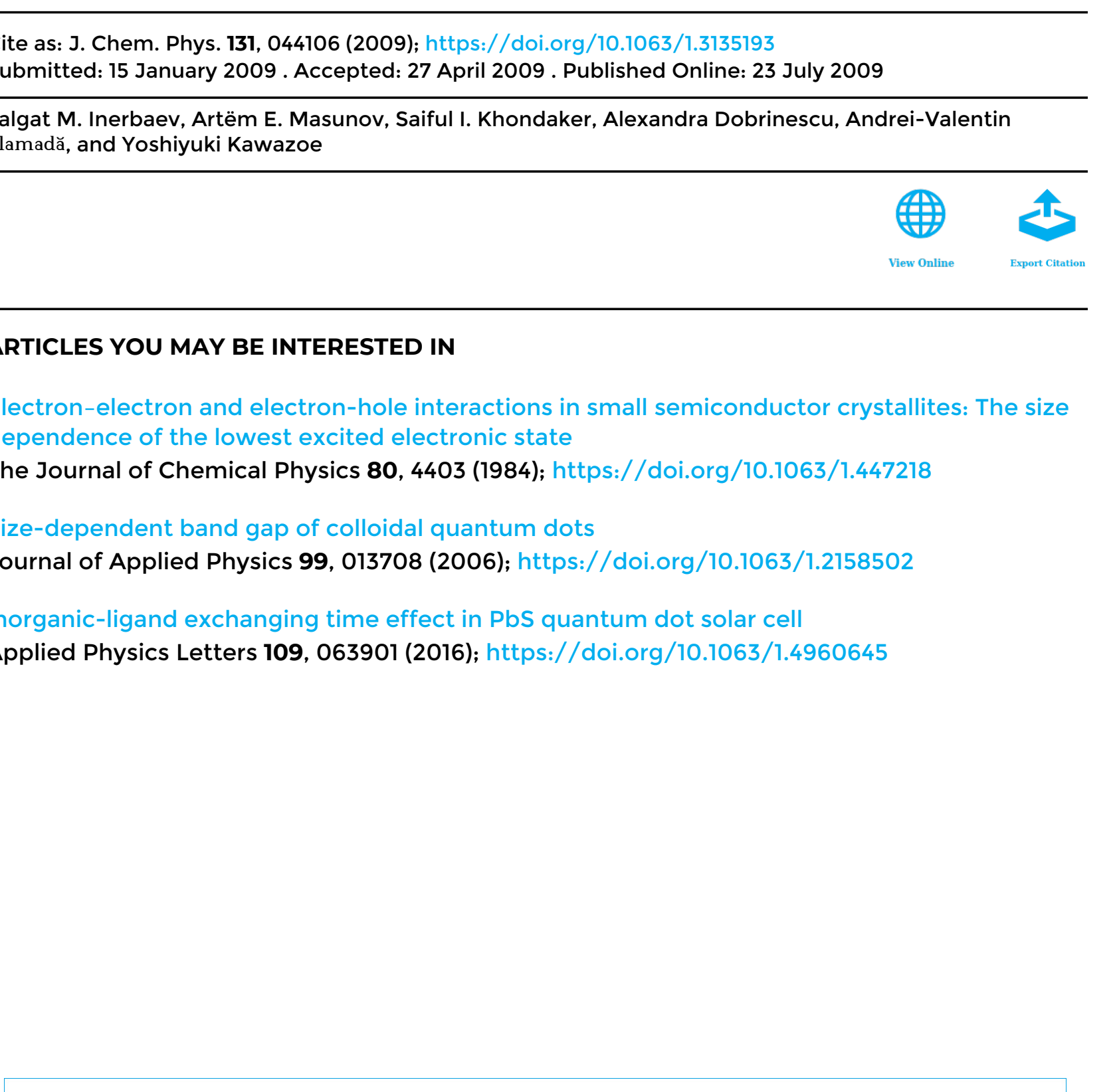

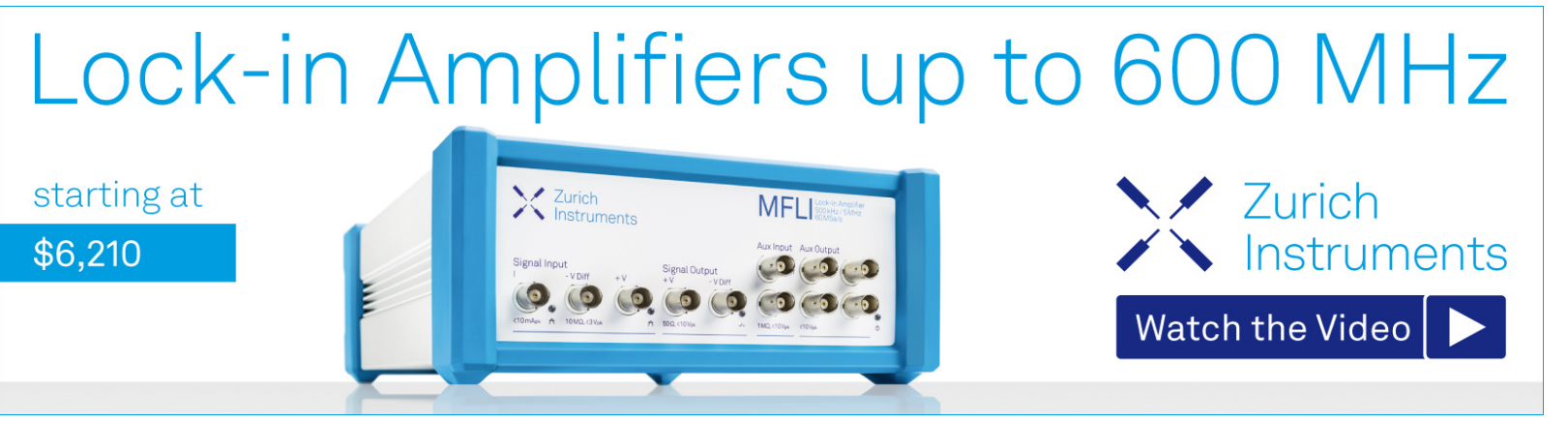

J. Chem. Phys. 131, 044106 (2009); https://doi.org/10.1063/1.3135193 


\title{
Quantum chemistry of quantum dots: Effects of ligands and oxidation
}

\author{
Talgat M. Inerbaev, ${ }^{1,2,5}$ Artëm E. Masunov, ${ }^{1,3,4, a)}$ Saiful I. Khondaker, ${ }^{1,4}$ \\ Alexandra Dobrinescu, ${ }^{1,6}$ Andrei-Valentin Plamadă, ${ }^{1,6}$ and Yoshiyuki Kawazoe ${ }^{5}$ \\ ${ }^{1}$ Nanoscience Technology Center, University of Central Florida, Orlando, Florida 32826, USA \\ ${ }^{2}$ Institute for Simulation and Training, University of Central Florida, Orlando, Florida 32826, USA \\ ${ }^{3}$ Department of Chemistry, University of Central Florida, Orlando, Florida 32826, USA \\ ${ }^{4}$ Department of Physics, University of Central Florida, Orlando, Florida 32826, USA \\ ${ }^{5}$ Institute for Materials Research, Tohoku University, Sendai 980-8577, Japan \\ ${ }^{6}$ Department of Physics, Alexandru Ioan Cuza University, Iasi 700506, Romania
}

(Received 15 January 2009; accepted 27 April 2009; published online 23 July 2009)

\begin{abstract}
We report Gaussian basis set density functional theory (DFT) calculations of the structure and spectra of several colloidal quantum dots $(\mathrm{QDs})$ with a $(\mathrm{CdSe})_{n}$ core $(n=6,15,17)$, that are either passivated by trimethylphosphine oxide ligands, or unpassivated and oxidized. From the ground state geometry optimization results we conclude that trimethylphosphine oxide ligands preserve the wurtzite structure of the QDs. Evaporation of the ligands may lead to surface reconstruction. We found that the number of two-coordinated atoms on the nanoparticle's surface is the critical parameter defining the optical absorption properties. For $(\mathrm{CdSe})_{15}$ wurtzite-derived QD this number is maximal among all considered QDs and the optical absorption spectrum is strongly redshifted compared to QDs with threefold coordinated surface atoms. According to the time-dependent DFT results, surface reconstruction is accompanied by a significant decrease in the linear absorption. Oxidation of QDs destroys the perfection of the QD surface, increases the number of two-coordinated atoms and results in the appearance of an infrared absorption peak close to $700 \mathrm{~nm}$. The vacant orbitals responsible for this near infrared transition have strong $\mathrm{Se}-\mathrm{O}$ antibonding character. Conclusions of this study may be used in optimization of engineered nanoparticles for photodetectors and photovoltaic devices. () 2009 American Institute of Physics.
\end{abstract}

[DOI: $10.1063 / 1.3135193$ ]

\section{INTRODUCTION}

Colloidal quantum dots (QDs) are chemically stable particles of nanometer size, intermediate between crystal and free molecule. Their fundamental electronic and optical properties are critically size dependent due to the quantum confinement effect. ${ }^{1}$ Many of the applications of colloidal QDs involve charge transport. Quantization of charge and energy resulting from quantum confinement is analogous with artificial atoms. Recent progress in nanotechnology allows the preparation of large arrays of these artificial atoms in which particle size, interparticle separation, and chemical composition are controlled, ${ }^{2}$ thus creating nanostructured solids with tunable properties.

Highly monodisperse QDs synthesized by convenient methods of organometallic chemistry are composed of a nanocluster of semiconducting material (core) covered on the surface with a monolayer of organic molecules (known as passivating ligands). ${ }^{3}$ Examples of these surface ligands are trioctylphosphine (TOP), TOP oxide (TOPO), and various amines. These ligands not only prevent agglomeration of the QDs during the synthesis process and solubilize them in various solvents but may also play a decisive role in stabilizing the structure of QDs and affect their electronic and optical properties (including the fine structure of band edge lumines-

\footnotetext{
a) Author to whom correspondence should be addressed. Electronic mail: amasunov@mail.ucf.edu.
}

cence). Among these ligands, TOPO is the most widely used, while amines usually give the strongest luminescence but easily dissociate. ${ }^{4}$ Surface ligands were also reported to affect the growth rates and to terminate the dangling bonds on the QD's surface. ${ }^{5}$ Binding of a single octadecanethiol molecule to a CdSe nanocrystal can decrease the photoluminescence quantum yield of that single nanocrystal by at least $50 \%$.

Reconstruction of the surface may also have a critical effect on optical properties of quantum dots. ${ }^{7}$ A recent survey showed that both the type and the quality of surface passivation are very important for the optical gain and light absorption. ${ }^{8}$ The surface structure of the QD nanocrystals is, however, not very well understood, and only limited experimental evidence is available. It had been established that the coordination of TOP to the Se atoms on the surface of CdSe QDs is responsible for the emission band that is redshifted relative to the photoluminescence band edge. ${ }^{9}$ On the other hand, for CdSe QDs capped by the oxygen-coordinating ligand TOPO experimental studies using NMR (Ref. 10) and $\mathrm{x}$-ray photoelectron spectroscopy ${ }^{11}$ techniques established that the surface Se atoms are not coordinated by TOPO. A similar effect was found for other ligands using extended $\mathrm{x}$-ray absorption fine structure. ${ }^{12}$

Accurate theoretical description of the electronic structure as well as optical and electron transport properties of QDs is critically important for the rational design of nano- 
structured solids. Simplistic particle-in-a-box models, also called effective-mass approximation, neglects the passivation, while focusing on modification of the electron envelope functions induced by the confinement. ${ }^{13}$ Unfortunately, it was found to give insufficient accuracy for small particle sizes. ${ }^{14}$ More accurate studies have been carried out at the atomistic scale, using semiempirical ${ }^{15}$ and density functional theory ${ }^{16}$ (DFT) levels. A ligand pseudopotential model has been used to simulate the surface passivation. ${ }^{17,18}$ In another study dangling bonds on the surface were removed by shifting the energies of the corresponding hybrid orbitals by about $100 \mathrm{eV}^{19}$ which is well above the conduction band edge. In several studies that combined a self-consistent reaction field method and semiempirical pseudopotential methods the electronic properties of CdSe nanocrystals were also found to be sensitive to their environment. ${ }^{20,21}$ However, the ambiguities in magnitude and location of the surface potential may have affected the calculated values of the optical gaps.

More sophisticated models explicitly include the ligands in atomistic simulations. Pseudo-hydrogen atoms are often used as model ligands in order to saturate dangling bonds. ${ }^{22}$ Recent QD calculations using the effective tight binding model and a very simple model for QD surface reconstruction based on observations of bulk surface relaxations were carried out using oxygen atoms as passivation ligands attached to surface $\mathrm{Cd}$ atoms. ${ }^{18}$ More realistic simulations including surface reconstruction effects using first principle methods, were published recently. ${ }^{7,21,23,24}$ These simulations predict self-healing ligand effects on CdSe nanocrystals.

Photovoltaic applications of colloidal QDs involve charge transport, and recent experiments have demonstrated the important role that charges play in optical properties of QDs. Although dark conductance of undoped CdSe QDs arrays was found to be extremely small, ${ }^{25,26}$ photoinduced conductivity has been observed. Measurements of the photocurrent response have shown that the photocurrent action spectra follow the light absorption spectra of the nanoparticles. ${ }^{25}$ The observation of extremely long-lived current transients suggests the importance of long-range Coulomb interactions between charges on different nanocrystals. ${ }^{26}$ In these experiments the inter-QD separation was determined by the length of organic capping molecules. Removal of these ligands from the QD's surface results in the decrease in both physical separation and tunneling barrier between QDs and increases the electrical conductivity through this artificial solid. There are two ways to remove the ligands: (i) anneal the QD solid at a temperature below the melting point of QD semiconductor core, and (ii) treat it chemically. ${ }^{27}$

One of the easiest methods of chemical treatment is oxidation. Unfortunately, exposure of samples to oxygen in air is difficult to control sometimes, thus the effect of the oxidation can be mistaken for the intrinsic properties of pristine QDs. For instance the electroluminescence for $2.5 \mathrm{~nm} \mathrm{CdSe}$ QDs was recently reported at two wavelengths: 650 and 490 $\mathrm{nm} .^{28}$ Clearly, $650 \mathrm{~nm}$ corresponds to a band gap $(1.9 \mathrm{eV})$ that is too narrow for strong quantum confinement in a QD that small, and is much closer to the bulk band gap. More controlled experiments for nanocrystalline thin films show, ${ }^{29}$ that increased density of oxygen is, in fact, the reason that leads to the defect states appearing $0.6 \mathrm{eV}$ below the band gap.

In the present contribution we discuss possible mechanisms that could explain such band gap narrowing. Our arguments are applicable to the array of weakly interconnected QDs with hopping electron conductivity between them. We report changes in electronic spectra of QDs due to effects of surface passivation by organic ligands and reconstruction of the nanoparticle surface after ligand removal. Surface passivation by TOPO ligands is found to stabilize QDs in bulklike hexagonal structure. Ligand removal results in a red shift the adsorption spectra and narrows the gap between the highest occupied molecular orbital (HOMO) and the lowest unoccupied molecular orbital (LUMO). We also study the effects of chemical oxidation of CdSe QDs, but physiosorption of the oxygen molecules on surface defects is not included in the scope of the present work. We show that addition of an oxygen atom to a QD results in a structure modification that leads to the appearance of an infrared adsorption band in the electronic spectra. The theoretical study presented here can be verified in experiments by ligand removal in presence of oxygen. An experiment is in progress toward that end.

\section{COMPUTATIONAL DETAILS}

All theoretical calculations were performed using the GAUSSIAN 2003 suite of programs. ${ }^{30}$ We used the hybrid DFT functional dubbed Becke, three-parameter, Lee-Yang-Parr (B3LYP) ${ }^{31}$ which includes a fraction of Hartree-Fock exchange to reduce the self-interaction error. The Los Alamos double- $\zeta$ effective core potential (LANL2DZ) ${ }^{32}$ and the associated basis set were used. Among a number of different available theory levels, B3LYP/LANL2DZ as implemented in GAUSSIAN03 was shown to be the best compromise between efficiency and accuracy. ${ }^{33}$ In the present contribution, this level of theory was applied to the organic ligands as well the CdSe core. The linear response time-dependent DFT ansatz was used to predict the electronic spectra.

We selected neutral clusters with the common formula $(\mathrm{CdSe})_{n}$ and $n=6,15,17$ (termed QD6, QD15, and QD17 in the following). Clusters cut out of the wurtzite structure using different choices for the origin may have different topologies for the same cluster size. ${ }^{34}$ We cut the clusters out of the bulk wurtzite structure with a sphere, centered in the middle of the Cd-Se bond, tetrahedral cavity, and octahedral cavity. Among different clusters, the most stable isomer was chosen. Following Ref. 34, the QD17 cluster was built with its center in the midpoint of the $\mathrm{Cd}-\mathrm{Se}$ bond along the crystallographic direction $c$, while QD6 was built with the center in the octahedral cavity. These structures can be considered homologous to QD33 and QD34, which form the peaks on the gas-phase mass spectra and are theoretically predicted to have a nanodiamond structure. ${ }^{34(\mathrm{~b})}$ Unlike these nanodiamonds, QD17 has one formula unit as the diamond core, and QD6 has none. All selected wurtzite-derived QDs are shown in the top row of Fig. 1.

The TOPO ligand, often used in experiments, was mod- 

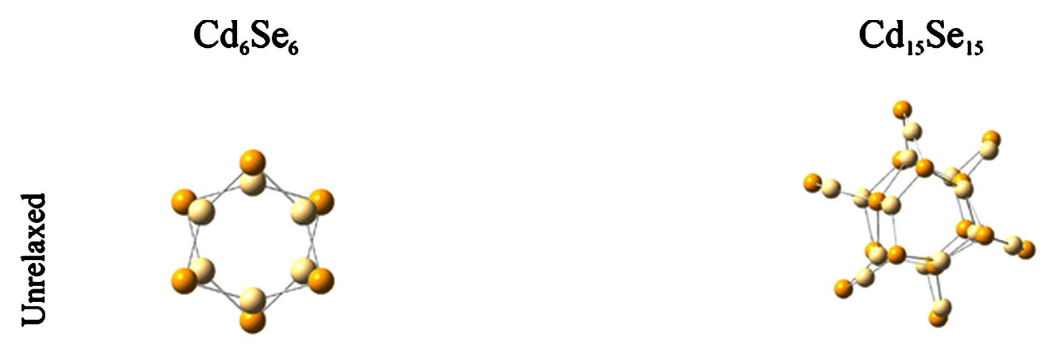

(a)
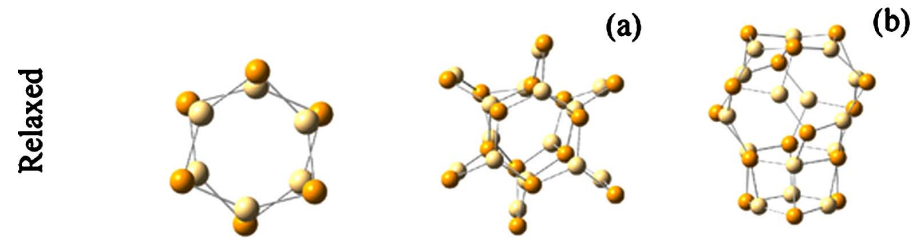

(b)

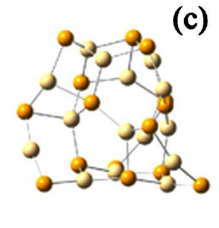

(c)

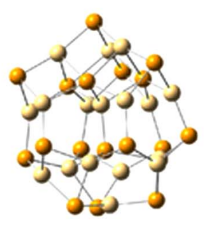

(e)

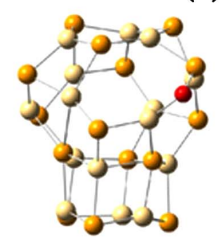

$\mathrm{Cd}_{17} \mathrm{Se}_{17}$

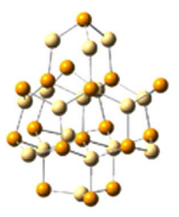

(d)

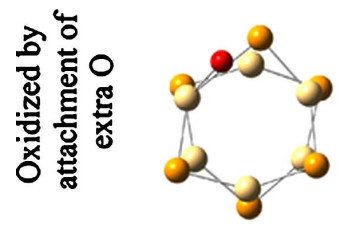

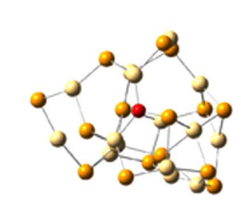
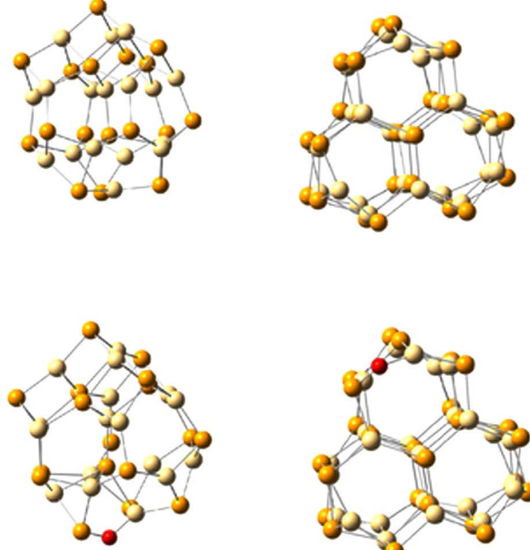

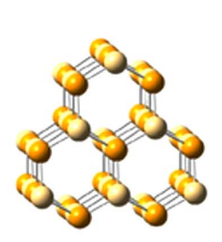

$\mathrm{Cd}_{26} \mathrm{Se}_{26}$

FIG. 1. The three rows correspond to unrelaxed, relaxed, and oxidized by attachment of extra oxygen atom wurtzite structures of $(\mathrm{CdSe})_{n}, n=6,15$, and 17 . For $n=15$ three different cases for relaxed QDs are presented: (a) is relaxed wurtzite-derived QD while (b) and (c) were obtained by reoptimization of oxidized structures (d) and (e) after detachment of oxygen atom. QDs (d) and (e) were obtained by attachment of extra oxygen to three- and two-coordinated Cd atoms, respectively. Structure (b) could also be obtained by sequential substitution of Se atom by $\mathrm{O}$ and backward with structure relaxation on each step. Cd is white, $\mathrm{Se}$ is yellow, and $\mathrm{O}$ is red. The optimized ligated structures cannot be clearly seen because of the hindering effect of the ligands. After the ligands are removed for clarity, they are indistinguishable from unrelaxed structures shown in the top row.

eled by trimethylphosphine oxide $\mathrm{OPMe}_{3}$. One or two ligands were attached to the surface $\mathrm{Cd}$ atoms, to complete their tetrahedral coordination, while the surface Se atoms were not capped with ligands. This is in agreement with other theoretical and experimental findings. Puzder et al. ${ }^{21}$ previously showed that the dominant binding interaction is between $\mathrm{O}$ atom of phosphine oxide and $\mathrm{Cd}$ atoms on the QD surface. Experimental observations ${ }^{35}$ also reveal much stronger interaction of oxygen- and sulfur-based ligands (TOPO, thiophenol, toluenethiol, and p-hydroxythiophenol) with $\mathrm{Cd}$ atoms than with $\mathrm{Se}$ atoms of QD surface.

\section{RESULTS AND DISCUSSION}

We investigated (i) the ligated forms of QDs, (ii) their unligated forms, (iii) forms with extra oxygen atom attached to the surface $\mathrm{Cd}$ atom, and (iv) forms where $\mathrm{Se}$ atom is replaced with the $\mathrm{O}$ atom. In each case, the geometry was optimized to the lowest energy configuration, absorption spectra were predicted, and spectral differences between the forms were analyzed.

There is one fundamental difference between QD6 and QD17, on one hand, and QD15, on the other hand. The former two clusters have only three-coordinated surface atoms in their relaxed structures, while QD15 has twocoordinated atoms. The stable closed-shell atomic structures display large bandgaps of 3.14 and $2.66 \mathrm{eV}$ for QD6 and QD17, respectively. In contrast, $(\mathrm{CdSe})_{15}$ has 12 twofold coordinated, or "defect," surface atoms and a narrow bandgap of $1.89 \mathrm{eV}$. This difference is reflected in the optical properties by a significant redshift of the QD15 linear absorption spectrum relative to the other clusters, as shown in Fig. 2.

From comparing the top row in the Fig. 1 one can see that the topology of QD6 and QD15 does not change with geometry relaxation. This is in agreement with results in Ref. 7. After relaxation, the geometry change of the ligated QDs is found to be even less than nonligated QDs. In fact, relaxed ligated geometries look identical to the unrelaxed wurzitederived ones, after the ligand is removed for clarity (not shown). Similarly, surface passivation by trimethylphosphine oxide ligands prevents surface relaxation and preserves the structure of nonrelaxed QD17. Thus, the topology of the ligated wurtzite-derived $\mathrm{CdSe}$ core remains the same as the nonrelaxed one. However, bare QD17 does relax somewhat from its wurzite-derived structure to reduce the number of two-coordinated Se atoms from 4 to 2 (Fig. 1). Passivation also leads to a notable blueshift in the adsorption spectra of all considered QDs. Bandgaps become wider and are equal to 4.03, 2.73, and $2.54 \mathrm{eV}$ for ligated wurtzite-derived QD6, QD15, and QD17, respectively.

Next we investigated QD oxidation effects by relaxing the geometry after (i) addition of the oxygen atom to the $\mathrm{Cd}$ atom on the surface of the QD, and (ii) substitution of Se atom with $\mathrm{O}$ atom. We observe that for QD6 and QD17 the additional oxygen atom is inserted into the $\mathrm{Cd}-\mathrm{Se}$ bond on the surface, instead of penetrating into the interior of QD 


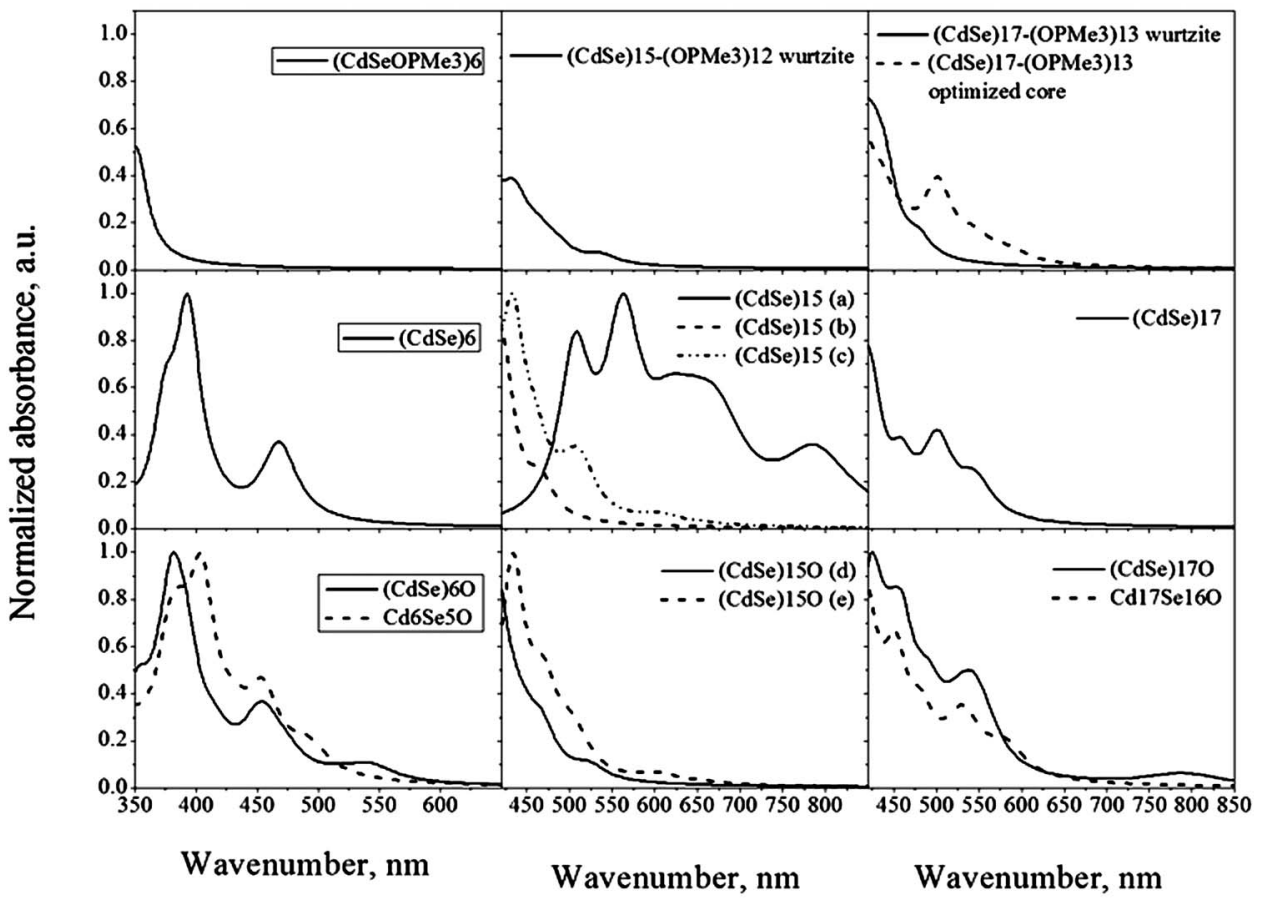

FIG. 2. Linear absorption spectra for unligated, oxidized, and ligated cadmium selenide QDs. For the closed atomic shell structures QD6 and QD17, the removal of the ligands does not change the absorption spectra, while oxidation generates an absorption band close to $800 \mathrm{~nm}$. The unstable unligated isomer QD15a reveals absorption spectrum strongly shifted into the infrared region. Oxidation leads to the reconstruction of QD's structure and a blueshift in adsorption spectra for QD15 $d$ and QD15e. Reduction in oxidized nanoparticles is accompanied by further blueshift for QD15b and QD15c relative to the QD with similar topology. Saturation of the dangling bonds of wurtzite-derived QD15 by ligands stabilizes it with calculated absorption spectrum close to the most stable modification QD15 $b$ with threefold coordinated surface atoms.

(bottom row in Fig. 1). This insertion increases Cd-Se distance by $0.8 \mathrm{~A}$, and is accompanied by minor distortion of QDs.

In sharp contrast, addition of an oxygen atom to QD15 leads to significant reconstruction. Attachment of an extra oxygen to a three-coordinated $\mathrm{Cd}$ atom leads its insertion into the $\mathrm{Cd}-\mathrm{Se}$ bond again, but in this case wurtzite-derived QD15a relaxes to distinctly different configuration QD15d (both shown in Fig. 1). This QD15d has an almost perfect surface with no two-coordinated surface atoms. At the same time, oxidation by attachment of an $\mathrm{O}$ atom to twocoordinated $\mathrm{Cd}$ leads to its coordination with two $\mathrm{Cd}$ and one Se atom, and formation of highly distorted configuration QD15e, shown in Fig. 1. This structure has several twocoordinated Se atoms on the surface, similar to QD15a. However, unlike QD15a, QD15e does not include twocoordinated $\mathrm{Cd}$ atoms.

In order to investigate if oxidation of QD15 is indeed irreversible, we removed the additional oxygen atom from QD15d, and reoptimized the geometry. As a result, a new isomer QD15b was obtained (Fig. 1). It can be described as cagelike polyhedron with 24 vertices and 10 facets (six hexagonal and four quadrangle ones) with one extra hexagon stacked on top of the hexagonal facet. The reduction in QD15e structure resulted in QD15c with four twocoordinated Se atoms on the surface. The relative energies of QD15a, QD15b, and QD15c are 4.92, 0.0, and $2.50 \mathrm{eV}$, respectively. The calculated binding energy per CdSe formula unit increases from 3.14 for QD15a to $3.47 \mathrm{eV}$ for the most stable QD15b.
As an alternate oxidation route we investigated substitution of selenium atom by oxygen. In the case of QD15a, this substitution resulted in a QD15b homolog. The substitution of $\mathrm{O}$ atom back to Se atom converges to the QD15b. We can speculate that high transition barrier between wurzitederived QD15a and more stable configuration QD15b can be bypassed by sequential oxidation and reduction processes. In the cases of substitutional oxidation of QD17 there are two possible positions for the oxygen atom: on the surface and inside the QD. Comparing ground state energies of the $\mathrm{Cd}_{17} \mathrm{Se}_{16} \mathrm{O}$ cluster demonstrates that the QD with the oxygen atom inside is $0.54 \mathrm{eV}$ more stable than the surface substituted one.

The theoretical absorption spectra of nanoclusters are presented in Fig. 2. Nonoxidized QD15 isomers were found to exhibit a blueshift on going from less to the more stable isomer. The wavelength for the first bright excited state decreased from $795 \mathrm{~nm}$ for the least stable wurtzite-derived conformation QD15a to $468 \mathrm{~nm}$ for the most stable QD15b. Comparison of adsorption spectra of QDs of different configurations clarifies the effect of dangling bonds on the optical properties of QDs. The adsorption spectrum for wurtzitederived structure QD15a is the most redshifted one among the QDs with the same stoichiometry, while decreasing the number of two-coordinated surface atoms moves the optical adsorption to the blue. The same trend holds for oxidized species: each considered QD reveals the appearance of an additional peak in the low frequency region after the attachment of the oxygen atom that breaks perfection or, in other 


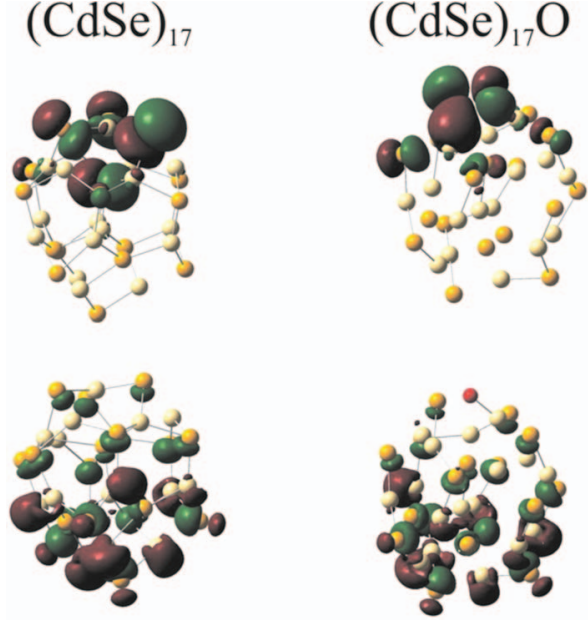

FIG. 3. Contour plots calculated for the highest occupied (bottom) and lowest unoccupied (top) one-electron states in $(\mathrm{CdSe})_{17}$ (left) and $(\mathrm{CdSe})_{17} \mathrm{O}$ (right) clusters. The unoccupied state in $(\mathrm{CdSe})_{17} \mathrm{O}$ has antibonding character with respect to the oxygen-selenium interaction. It narrows the bandgap to the value of $1.923 \mathrm{vs} 2.661 \mathrm{eV}$ bandgap for $(\mathrm{CdSe})_{17}$. Energy levels for HOMO are equal to -6.257 and $-5.569 \mathrm{eV}$, while for LUMO they are $-3.596 \mathrm{eV}$ and $-3.646 \mathrm{eV}$ for $(\mathrm{CdSe})_{17}$ and $(\mathrm{CdSe})_{17} \mathrm{O}$ QDs, respectively.

words, threefold coordination of the surface structure. We conclude that the number of surface "defects" controls the optical adsorption energy range.

To understand the nature of oxidation effect on the optical spectra, we plotted in Fig. 3 the isosurfaces of the KohnSham orbitals involved in the lowest excited states for both oxidized and nonoxidized clusters. For all considered QDs the effect of inserted oxygen atom is qualitatively similar, so only the results for QD17 are presented in Fig. 3. Compared to the nonoxidized QD17 cluster, the oxidized one has one unoccupied level inside the HOMO-LUMO gap which corresponds to an antibonding $\mathrm{Se}-\mathrm{O}$ orbital.

\section{CONCLUSIONS}

We studied the geometry relaxation and linear absorption spectra of cadmium selenide QDs with different surface topologies. We found that the number of two-coordinated atoms on the nanoparticle's surface is the critical parameter defining the optical absorption properties. For $(\mathrm{CdSe})_{15}$ wurtzite-derived QD this number is maximal among all considered QDs and the optical absorption spectrum is strongly shifted into the infrared region relative to other QDs with threefold coordinated surface atoms. The surface reconstruction may decrease the number of two-coordinated atoms and leads to the blueshift in absorption spectra of bare QDs. Passivation of the surface with organic ligands stabilizes the wurtzite structure of QDs, prevents this surface relaxation, and leads to further blueshift in absorption spectra relative to the bare QDs. Oxidation of QDs destroys the perfection of the QD surface, increases the number of two-coordinated atoms and results in appearance of an infrared adsorption band. This study may be used in optimization of nanomaterials for photodetector and photovoltaic devices.

\section{ACKNOWLEDGMENTS}

Research was performed in part using (1) Stokes HPCC facility at UCF Institute for Simulation and Training (IST), (2) Bethe SMP server at UCF NanoScience Technology Center (NSTC), (3) the National Energy Research Scientific Computing Center (NERSC), a DOE Office of Science user facility at Lawrence Berkeley National Laboratory (4) SR11000-K2/51 supercomputing system supported by the Information Science Group of the Institute for Materials Research, Tohoku University. A.D. and A.-V.P. acknowledge support by the NSTC International Internship program.

${ }^{1}$ W. J. Parak, D. Gerion, T. Pellegrino, D. Zanchet, C. Micheel, S. C. Williams, R. Boudreau, M. A. Le Gros, C. A. Larabell, and A. P. Alivisatos, Nanotechnology 14, R15 (2003); X. Michalet, F. Pinaud, T. D. Lacoste, M. Dahan, M. P. Bruchez, A. P. Alivisatos, and S. Weiss, Single Mol. 2, 261 (2001).

${ }^{2}$ C. B. Murray, C. R. Kagan, and M. G. Bawendi, Annu. Rev. Mater. Sci. 30, 545 (2000).

${ }^{3}$ C. B. Murray, D. J. Norris, and M. G. Bawendi, J. Am. Chem. Soc. 115, 8706 (1993).

${ }^{4}$ A. V. Malko, A. A. Mikhailovsky, M. A. Petruska, J. A. Hollingsworth, and V. I. Klimov, J. Phys. Chem. B 108, 5250 (2004).

${ }^{5}$ X. G. Peng, L. Manna, W. D. Yang, J. Wickham, E. Scher, A. Kadavanich, and A. P. Alivisatos, Nature (London) 404, 59 (2000); L. Manna, E. C. Scher, and A. P. Alivisatos, J. Am. Chem. Soc. 122, 12700 (2000); Z. A. Peng and X. G. Peng, ibid. 124, 3343 (2002); Z. A. Peng and X. G. Peng, ibid. 123, 1389 (2001).

${ }^{6}$ A. M. Munro and D. S. Ginger, Nano Lett. 8, 2585 (2008).

${ }^{7}$ A. Puzder, A. J. Wlliamson, F. Gygi, and G. Galli, Phys. Rev. Lett. 92, 217401 (2004).

${ }^{8}$ J. A. McGuire, J. Joo, J. M. Pietryga, R. D. Schaller, and V. I. Klimov, Acc. Chem. Res. 41, 1810 (2008).

${ }^{9}$ G. Kalyuzhny and R. W. Murray, J. Phys. Chem. B 109, 7012 (2005).

${ }^{10}$ L. R. Becerra, C. B. Murray, G. G. Griffin, and M. G. Bavendi, J. Chem. Phys. 100, 3297 (1994).

${ }^{11}$ J. E. B. Katari, V. L. Colven, and A. P. Alivistos, J. Phys. Chem. 98, 4109 (1994)

${ }^{12}$ A. C. Carter, C. E. Bouldin, K. M. Kemmer, M. I. Bell, J. C. Woicik, and S. A. Majetich, Phys. Rev. B 55, 13822 (1997).

${ }^{13}$ H. Haug and S. W. Kock, Quantum Theory of the Optical and Electronic Properties of Semiconductors (World Scientific, Singapore, 1993).

${ }^{14}$ A. Zunger, Phys. Status Solidi B 224, 727 (2001); M. Califano and A. Zunger, Abstr. Pap. Am. Chem. Soc. 225, U452 (2003); L. W. Wang and A. Zunger, Phys. Rev. B 53, 9579 (1996).

${ }^{15}$ V. S. Gurin, J. Phys.: Condens. Matter 6, 8691 (1994); J. Robles, O. Mayorga, T. Lee, and D. Diaz, Nanostruct. Mater. 11, 283 (1999); K. Toth and T. A. Pakkanen, J. Comput. Chem. 14, 667 (1993).

${ }^{16}$ J. M. Matxain, J. E. Flowler, and J. M. Ugalde, Phys. Rev. A 61, 053201 (2000); J. O. Joswig, M. Springborg, and G. Seifert, J. Phys. Chem. B 104, 2617 (2000).

${ }^{17}$ C. Troparevsky, L. Kronik, and J. R. Chelikowsky, J. Chem. Phys. 114, (2001); 119, 2284 (2003); K. Leung and K. B. Whaley, J. Chem. Phys. 110, 11012 (1999).

${ }^{18}$ S. Pokrant and K. B. Whaley, Eur. Phys. J. D 6, 255 (1999).

${ }^{19}$ P. C. Chen and K. B. Whaley, Phys. Rev. B 70, 045311 (2004).

${ }^{20}$ T. Rabani, B. Hetényi, and B. Berne, J. Chem. Phys. 110, 5355 (1999); J. T. Hu, L. W. Wang, L. S. Li, W. D. Yang, and A. P. Alivisatos, J. Phys. Chem. B 106, 2447 (2002); J. R. Sachleben, V. Colvin, L. Emsley, E. W. Wooten, and A. P. Alivisatos, ibid. 102, 10117 (1998); D. J. Milliron, A. P. Alivisatos, C. Pitois, C. Edder, and J. M. J. Frechet, Adv. Mater. (Weinheim, Ger.) 15, 58 (2003); L. Manna, L. W. Wang, R. Cingolani, and A. P. Alivisatos, J. Phys. Chem. B 109, 6183 (2005).

${ }^{21}$ A. Puzder, A. J. Williamson, N. Zaitseva, G. Galli, G. Manna, and A. P. Alivisatos, Nano Lett. 4, 2361 (2004)

${ }^{22}$ M. L. del Puerto, M. L. Tiago, and J. R. Chelikowsky, Phys. Rev. Lett. 97, 096401 (2006); E. Badaeva, Y. Feng, D. R. Gamelin, and X. LiNew J. Phys. 10, 055013 (2008).

${ }^{23}$ S. Kilina, S. Ivanov, and S. Tretiak, J. Am. Chem. Soc. 131, 7717 (2008).

${ }^{24}$ L. Pizzagalli, G. Galli, J. E. Klepeis, and F. Gygi, Phys. Rev. B 63, (2001); H. Kamisaka, S. V. Kilina, K. Yamashita, and O. V. Prezhdo, J. 
Phys. Chem. C 112, 7800 (2008).

${ }^{25}$ D. S. Ginger and N. C. Greenham, J. Appl. Phys. 87, 1361 (2000).

${ }^{26}$ N. Y. Morgan, C. A. Leatherdale, M. Drndic, M. V. Jarosz, M. A. Kastner, and M. Bawendi, Phys. Rev. B 66, 075339 (2002).

${ }^{27}$ V. J. Porter, S. Geyer, J. E. Halpert, M. A. Kastner, and M. G. Bawendi, J. Phys. Chem. C 112, 2308 (2008).

${ }^{28}$ J. G. Zhou, J. Zhu, J. Brzezinski, and Z. F. Ding, Can. J. Chem. 87, 386 (2009).

${ }^{29}$ Z. Aneva, D. Nesheva, C. Main, S. Reynolds, A. G. Fitzgerald, and E. Vateva, Semicond. Sci. Technol. 23, 095002 (2008).

${ }^{30}$ M. J. Frisch, G. W. Trucks, H. B. Schlegel et al., Gaussian 03, Revision C.02 (Gaussian, Inc., Wallingford CT, 2004).

${ }^{31}$ A. D. Becke, J. Chem. Phys. 98, 5648 (1993); C. T. Lee, W. T. Yang, and R. G. Parr, Phys. Rev. B 37, 785 (1988).

${ }^{32}$ P. J. Hay and R. W. Wadt, J. Chem. Phys. 82, 270 (1985); R. W. Wadt and P. J. Hay, ibid. 82, 284 (1985); P. J. Hay and R. W. Wadt, ibid. 82, 299 (1985).

${ }^{33}$ P. Yang, S. Tretiak, A. E. Masunov, and S. Ivanov, J. Chem. Phys. 129, 12 (2008).

${ }^{34}$ M. Yu, G. W. Fernando, R. Li, F. Papadimitrakopoulos, N. Shi, and R. Ramprasad, Appl. Phys. Lett. 88, 222903 (2006); A. Kasuya, Y. Noda, I. Dmitruk, V. Romanyuk, Y. Barnakov, K. Tohji, V. Kumar, R. Belosludov, Y. Kawazoe, and N. Ohuchi, Eur. Phys. J. D 34, 39 (2005); A. Kasuya, R. Sivamohan, Y. A. Barnakov, I. M. Dmitruk, T. Nirasawa, V. R. Romanyuk, V. Kumar, S. V. Mamykin, K. Tohji, B. Jeyadevan, K. Shinoda, T. Kudo, O. Terasaki, Z. Liu, R. V. Belosludov, V. Sundararajan, and Y. Kawazoe, Nature Materials 3, 99 (2004).

${ }^{35}$ I. S. Liu, H. H. Lo, C. T. Chien, Y. Y. Lin, C. W. Chen, Y. F. Chen, W. F. $\mathrm{Su}$, and S. C. Liou, J. Mater. Chem. 18, 675 (2008). 